\title{
Periodic Solutions for Circular Restricted $N+1$-Body Problems
}

\author{
Xiaoxiao Zhao, ${ }^{1}$ Wei $\mathrm{Li}^{2}$ and Peng Wang ${ }^{1}$ \\ ${ }^{1}$ College of Mathematics, Sichuan University, Chengdu 610064, China \\ ${ }^{2}$ School of Economic Mathematics, Southwestern University of Finance and Economics, Chengdu 610074, China
}

Correspondence should be addressed to Xiaoxiao Zhao; zxgg2007@163.com

Received 18 February 2013; Accepted 3 April 2013

Academic Editor: Baodong Zheng

Copyright (C) 2013 Xiaoxiao Zhao et al. This is an open access article distributed under the Creative Commons Attribution License, which permits unrestricted use, distribution, and reproduction in any medium, provided the original work is properly cited.

For circular restricted $N+1$-body problems, we study the motion of a sufficiently small mass point (called the zero mass point) in the plane of $N$ equal masses located at the vertices of a regular polygon. By using variational minimizing methods, for some $N$, we prove the existence of the noncollision periodic solution for the zero mass point with some fixed wingding number.

\section{Introduction and Main Results}

In this paper, we study the planar circular restricted $N+1$ body problems. Suppose that points of positive masses $m_{1}=$ $\cdots=m_{N}=1$ move on a circular orbit around the center of masses, and that the sufficiently small mass point, called the zero mass point, moves on the moving plane of the given $N$ equal masses and does not influence the motion of $m_{1}, \ldots, m_{N}$, but the motion of the zero mass point is affected by the given $N$ equal mass points. Let $\rho_{i}=e^{\sqrt{-1}(2 \pi i / N)}$ and we denote the position vectors of the given $N$ bodies by $q_{i}(t)$, $i=1, \ldots, N$, then

$$
\begin{gathered}
q_{1}(t)=r e^{\sqrt{-1} 2 \pi t} \rho_{1}, \ldots, \quad q_{i}(t)=r e^{\sqrt{-1} 2 \pi t} \rho_{i}, \ldots, \\
q_{N}(t)=r e^{\sqrt{-1} 2 \pi t},
\end{gathered}
$$

where the radius $r>0$. It is known that $q_{1}(t), \ldots, q_{N}(t)$ satisfy the following Newtonian equations:

$$
m_{i} \ddot{q}_{i}=\frac{\partial U}{\partial q_{i}}, \quad i=1, \ldots, N,
$$

where

$$
U=\sum_{1 \leq i<j \leq N} \frac{m_{i} m_{j}}{\left|q_{i}-q_{j}\right|} .
$$

We also assume that

$$
\sum_{i=1}^{N} m_{i} q_{i}=\sum_{i=1}^{N} q_{i}=0
$$

The orbit $q(t) \in R^{2}$ for the zero mass point is governed by the gravitational forces of $m_{1}, \ldots, m_{N}$ and therefore it satisfies the following equation:

$$
\ddot{q}=\sum_{i=1}^{N} \frac{m_{i}\left(q_{i}-q\right)}{\left|q_{i}-q\right|^{3}} .
$$

For $N$-body problems, there are many papers concerned with the periodic solutions by using variational methods; see [1-15] and the references therein. In [1], Chenciner and Montgomery proved the existence of the remarkable figure"8" type periodic solution for planar Newtonian 3-body problems with equal masses. Marchal [4] studied the fixed end problem for Newtonian $n$-body problems and proved that the minimizer for the Lagrangian action has no interior collision. In [6], Simó used computer to discover many new periodic solutions for Newtonian $n$-body problems. Zhang and Zhou [10-12] decomposed the Lagrangian action for $n$ body problems into some sum for two body problems and $[11,12]$ avoided collisions by comparing the lower bound for the Lagrangian action on the symmetry collision orbits and the upper bound for the Lagrangian action on test orbits in some cases.

Motivated by the above works, we use variational methods to study the circular restricted $N+1$-body problem with some fixed wingding numbers and $N$ equal masses.

For the readers' conveniences, we recall the definition of the winding number, which can be found in many books on the classical differential geometry. 
Definition 1. Let $\Gamma: x(t), t \in[a, b]$ be a given oriented continuous closed curve, and $p$ be a point on the plane not on the curve. Then, the mapping $\varphi: \Gamma \rightarrow S^{1}$ given by

$$
\varphi(x(t))=\frac{x(t)-p}{|x(t)-p|}, \quad t \in[a, b]
$$

is defined to be the position mapping of the curve $\Gamma$ relative to $p$, and when the point on $\Gamma$ goes around the curve once, its image point $\varphi(x(t))$ will go around $S^{1}$ a number of times; this number is called the winding number of the curve $\Gamma$ relative to $p$, and we denote it by $\operatorname{deg}(\Gamma, p)$. If $p$ is the origin, we write $\operatorname{deg} \Gamma$.

Let

$$
R(n)=\left(\begin{array}{cc}
\cos \frac{2 \pi}{n} & -\sin \frac{2 \pi}{n} \\
\sin \frac{2 \pi}{n} & \cos \frac{2 \pi}{n}
\end{array}\right) \in S O(2)
$$

be a counter-clockwise rotation of angle $2 \pi / n$ in $R^{2}$.
Define

$$
\begin{aligned}
W^{1,2} & \left(\frac{R}{Z}, R^{2}\right) \\
& =\left\{x(t) \mid x(t), \dot{x}(t) \in L^{2}\left(R, R^{2}\right), x(t+1)=x(t)\right\} .
\end{aligned}
$$

The norm of $W^{1,2}\left(R / Z, R^{2}\right)$ is

$$
\|x\|=\left[\int_{0}^{1}|x|^{2} d t\right]^{1 / 2}+\left[\int_{0}^{1}|\dot{x}|^{2} d t\right]^{1 / 2}
$$

We consider the Lagrangian functional of (5) as

$$
f(q)=\int_{0}^{1}\left[\frac{1}{2}|\dot{q}|^{2}+\sum_{i=1}^{N} \frac{1}{\left|q-q_{i}\right|}\right] d t
$$

on $\Lambda_{ \pm}$, where

$$
\begin{gathered}
\Lambda_{-}=\left\{q \in W^{1,2}\left(\frac{R}{Z}, R^{2}\right) \mid \begin{array}{r}
q\left(t+\frac{1}{2}\right)=R(2) q(t), q(t) \neq q_{i}(t), \forall t \in[0,1], i=1, \ldots, N, \\
\operatorname{deg}\left(q-q_{N}\right)=-1, \operatorname{deg}\left(q_{j}-q_{N}\right)=1, j=1, \ldots, N-1
\end{array}\right\}, \\
\Lambda_{+}=\left\{q \in W^{1,2}\left(\frac{R}{Z}, R^{2}\right) \mid \begin{array}{c}
q\left(t+\frac{1}{N}\right)=R(N) q(t), q(t) \neq q_{i}(t), \forall t \in[0,1], i=1, \ldots, N, \\
\operatorname{deg}\left(q-q_{N}\right)=1, \operatorname{deg}\left(q_{j}-q_{N}\right)=1, j=1, \ldots, N-1
\end{array}\right\} .
\end{gathered}
$$

Our main results are the following.

Theorem 2. For $N=2,3$, the minimizer of $f(q)$ on the closure $\bar{\Lambda}_{-}$of $\Lambda_{-}$is a noncollision 1-periodic solution of (5).

Remark 3. In proving Theorem 2, we need to use test functions. We find that when $N=3$, if the test functions are circular orbits, we cannot get the desired results on $\bar{\Lambda}_{-}$. Therefore, we select elliptic orbits as test functions.

Theorem 4. For $2 \leq N \leq 32402$, the minimizer of $f(q)$ on the closure $\bar{\Lambda}_{+}$of $\Lambda_{+}$is a noncollision 1-periodic solution of (5).

\section{Preliminaries}

In this section, we will list some basic lemmas and inequality to prove our theorems.

Lemma 5 (see [8]). The radius $r$ for the moving orbit of $N$ equal mass points is

$$
r=\left(\frac{1}{4 \pi}\right)^{2 / 3}\left[\sum_{1 \leq j \leq N-1} \csc \left(\frac{\pi}{N} j\right)\right]^{1 / 3} .
$$

Lemma 6 (Zhang and Zhou [9]). Suppose that $g \in S O(k)$ is an element of finite order $s$ and it has no fixed point other than the origin (i.e., 1 is not an eigenvalue of $g$ ). Then $\int_{0}^{T} X(t) d t=0$ for all $X(t)=\left(x_{1}(t), \ldots, x_{N}(t)\right) \in E_{g}^{N}$, where $E_{g}^{N}=\left\{\left(x_{1}, \ldots, x_{N}\right) \mid x_{i} \in H^{1}\left(R / T Z, R^{K}\right), x_{i}(t+T / s)=\right.$ $\left.g\left(x_{i}(t)\right), \forall t \in[0, T], i=1, \ldots, N\right\}$.

Lemma 7 (Poincare-Wirtinger inequality [16]). Let $q \in$ $W^{1,2}\left(R / T Z, R^{K}\right)$ and $\int_{0}^{T} q(t) d t=0$, and then

$$
\int_{0}^{T}|q(t)|^{2} d t \leq \frac{T^{2}}{4 \pi^{2}} \int_{0}^{T}|\dot{q}(t)|^{2} d t .
$$

Lemma 8 (see [17]). Let $X$ be a reflexive Banach space, $S$ be a weakly closed subset of $X$, and $f: S \rightarrow R \cup\{+\infty\}$. If $f \not \equiv+\infty$ is weakly lower semicontinuous and coercive $(f(x) \rightarrow+\infty$ as $\|x\| \rightarrow+\infty)$, then $f$ attains its infimum on $S$.

Lemma 9 (Palais's symmetry principle [18]). Let $\sigma$ be an orthogonal representation of a finite or compact group $G$, let $H$ be a real Hilbert space, and let $f: H \rightarrow R$ satisfy $f(\sigma \cdot x)=$ $f(x), \forall \sigma \in G, \forall x \in H$.

Set $F=\{x \in H \mid \sigma \cdot x=x, \forall \sigma \in G\}$. Then the critical point of $f$ in $F$ is also a critical point of $f$ in $H$.

Remark 10. By Palais's symmetry principle and the perturbation invariance for wingding numbers, we know that 
the critical point of $f(q)$ in $\Lambda_{ \pm}$is a periodic solution of Newtonian equation (5).

Lemma 11. (1) (Gordon's theorem [19]) Let $x \in$ $W^{1,2}\left(\left[t_{1}, t_{2}\right], R^{K}\right)$ and $x\left(t_{1}\right)=x\left(t_{2}\right)=0$. Then for any $a>0$, one has

$$
\int_{t_{1}}^{t_{2}}\left(\frac{1}{2}|\dot{x}|^{2}+\frac{a}{|x|}\right) d t \geq \frac{3}{2}(2 \pi)^{2 / 3} a^{2 / 3}\left(t_{2}-t_{1}\right)^{1 / 3}
$$

(2) (Long and Zhang [20]) Let $x \in W^{1,2}\left(R / T Z, R^{K}\right)$, and $\int_{0}^{T} x d t=0$, then for any $a>0$, we have

$$
\int_{0}^{T}\left(\frac{1}{2}|\dot{x}|^{2}+\frac{a}{|x|}\right) d t \geq \frac{3}{2}(2 \pi)^{2 / 3} a^{2 / 3} T^{1 / 3}
$$

\section{Proof of Theorems}

In order to get our theorems, we need two steps to complete the proof.

Step 1. We will establish the existence of variational minimizers of $f(q)$ in $(10)$ on $\bar{\Lambda}_{ \pm}$.

Lemma 12. $f(q)$ in (10) attains its infimum on $\bar{\Lambda}_{ \pm}$.

Proof. It is easy to check that the eigenvalue $\lambda$ of $R(n)$ satisfies

$$
\left\{\begin{array}{l}
\lambda=\cos \frac{2 \pi}{n} \\
\sin \frac{2 \pi}{n}=0
\end{array}\right.
$$

which implies $\lambda \neq 1$ for $n \geq 2$. Then by Lemma 6 , we have

$$
\int_{0}^{1} q(t)=0 \quad \forall q \in \bar{\Lambda}_{ \pm}
$$

So by using Lemma 7 , for all $q \in \bar{\Lambda}_{+}$, we see that an equivalent norm of (9) on $\bar{\Lambda}_{+}$is

$$
\|q\| \cong\left[\int_{0}^{T}|\dot{q}|^{2} d t\right]^{1 / 2}
$$

Hence, by the definition of $f(q), f$ is coercive on $\bar{\Lambda}_{ \pm}$.

Similar to the proof of Lemma 2.4 in [13], we can get the conclusion that $f$ is weakly lower semi-continuous on $\bar{\Lambda}_{ \pm}$.

Therefore, by using Lemma 8 , it can be concluded that $f(q)$ in (10) attains its infimum on $\bar{\Lambda}_{ \pm}$.

Step 2. We will prove that the variational minimizer in Lemma 12 is the noncollision 1-period solution of (5).

For any collision generalized solution $q$, we can estimate the lower bound for the value of Lagrangian action functional.
Lemma 13. For $\partial \Lambda_{-}=\left\{q \in W^{1,2}\left(R / Z, R^{2}\right) \mid q(t+1 / 2)=\right.$ $R(2) q(t), \exists 1 \leq i_{0} \leq N, t_{0} \in[0,1]$ s.t. $\left.q_{i_{0}}\left(t_{0}\right)=q\left(t_{0}\right)\right\}$, we have

$$
\begin{aligned}
& \inf _{q \in \partial \Lambda_{-}} f(q) \\
& \quad \geq \frac{3}{2}(4 \pi)^{2 / 3} N^{-1 / 3}+\left(\frac{N-1}{N}\right) \frac{3}{2}(2 \pi)^{2 / 3} N^{2 / 3}-\frac{1}{2}(2 \pi)^{2} r^{2} \\
& \triangleq d_{0} .
\end{aligned}
$$

Proof. It follows from (4) that

$$
\sum_{i=1}^{N} \dot{q}_{i}=0
$$

which implies

$$
\begin{aligned}
\sum_{i=1}^{N} \mid \dot{q} & -\left.\dot{q}_{i}\right|^{2} \\
& =\sum_{i=1}^{N}\left(|\dot{q}|^{2}+\left|\dot{q}_{i}\right|^{2}-2\left\langle\dot{q}, \dot{q}_{i}\right\rangle\right) \\
& =N|\dot{q}|^{2}+\sum_{i=1}^{N}\left|\dot{q}_{i}\right|^{2}-2\left\langle\dot{q}, \sum_{i=1}^{N} \dot{q}_{i}\right\rangle \\
& =N|\dot{q}|^{2}+\sum_{i=1}^{N}\left|\dot{q}_{i}\right|^{2} .
\end{aligned}
$$

Therefore,

$$
|\dot{q}|^{2}=\frac{1}{N} \sum_{i=1}^{N}\left(\left|\dot{q}-\dot{q}_{i}\right|^{2}-\left|\dot{q}_{i}\right|^{2}\right) .
$$

Hence,

$$
\begin{aligned}
f(q)= & \int_{0}^{1}\left[\frac{1}{2}|\dot{q}|^{2}+\sum_{i=1}^{N} \frac{1}{\left|q-q_{i}\right|}\right] d t \\
= & \frac{1}{N} \int_{0}^{1} \sum_{i=1}^{N}\left[\frac{1}{2}\left|\dot{q}-\dot{q}_{i}\right|^{2}+\frac{N}{\left|q-q_{i}\right|}\right] d t \\
& -\frac{1}{2 N} \int_{0}^{1} \sum_{i=1}^{N}\left|\dot{q}_{i}\right|^{2} d t .
\end{aligned}
$$

If $q \in \bar{\Lambda}_{-}$is a collision solution, then there exists $t_{0} \in[0,1]$ and $1 \leq i_{0} \leq N$ s.t $q\left(t_{0}\right)=q_{i_{0}}\left(t_{0}\right)$. Since $q_{i}(t+1 / 2)=R(2) q_{i}(t)$, one has $q\left(t_{0}+k / 2\right)=q_{i_{0}}\left(t_{0}+k / 2\right), \forall 0 \leq k \leq 2$. So, by (1) of Lemma 11, we get

$$
\begin{aligned}
& \frac{1}{N} \int_{0}^{1}\left[\frac{1}{2}\left|\dot{q}-\dot{q}_{i_{0}}\right|^{2}+\frac{N}{\left|q-q_{i_{0}}\right|}\right] d t \\
& \quad=\frac{2}{N} \int_{0}^{1 / 2}\left[\frac{1}{2}\left|\dot{q}-\dot{q}_{i_{0}}\right|^{2}+\frac{N}{\left|q-q_{i_{0}}\right|}\right] d t \\
& \quad \geq \frac{3}{2}(4 \pi)^{2 / 3} N^{-1 / 3} .
\end{aligned}
$$


For noncollision pair $q, q_{i}\left(i \neq i_{0}\right)$, we claim $\int_{0}^{1}(q(t)-$ $\left.q_{i}(t)\right) d t=0$. In fact, it follows from (1) that $q_{i+1}(t)=q_{i}(t+$ $1 / N)$, which implies

$$
q_{i}(t)=q_{1}\left(t+\frac{i-1}{N}\right)
$$

Therefore, by (4), we have

$$
\begin{aligned}
& \int_{0}^{1} q_{1}(t) d t \\
& \quad=\int_{0}^{1 / N} q_{1}(t) d t+\cdots+\int_{(N-1) / N}^{1} q_{1}(t) d t \\
& \quad=\int_{0}^{1 / N} q_{1}(t) d t+\cdots+\int_{0}^{1 / N} q_{1}\left(t+\frac{N-1}{N}\right) d t \\
& \quad=\int_{0}^{1 / N} \sum_{i=1}^{N} q_{i}(t) d t \\
& \quad=0 .
\end{aligned}
$$

Since $q_{1}(t+1)=q_{1}(t)$, by (25) and (26), we obtain

$$
\int_{0}^{1} q_{i}(t) d t=\int_{0}^{1} q_{1}\left(t+\frac{i-1}{N}\right) d t=\int_{0}^{1} q_{1}(t) d t=0
$$

Combined with (17) and (27), we have $\int_{0}^{1}\left(q(t)-q_{i}(t)\right) d t=0$. Hence, by (2) of Lemma 11, we can get

$$
\begin{gathered}
\frac{1}{N} \int_{0}^{1} \sum_{i \neq i_{0}}\left[\frac{1}{2}\left|\dot{q}-\dot{q}_{i}\right|^{2}+\frac{N}{\left|q-q_{i}\right|}\right] d t \\
\geq\left(\frac{N-1}{N}\right) \frac{3}{2}(2 \pi)^{2 / 3} N^{2 / 3} .
\end{gathered}
$$

For the other term of $f$, using the expression for the orbits $q_{1}, \ldots, q_{N}$ as in (1), one has

$$
-\frac{1}{2 N} \int_{0}^{1} \sum_{i=1}^{N}\left|\dot{q}_{i}\right|^{2} d t=-\frac{1}{2}(2 \pi)^{2} r^{2}
$$

Therefore, it follows from (24), (28), and (29) that

$$
\begin{aligned}
& \inf _{q \in \partial \Lambda_{-}} f(q) \\
& \quad \geq \frac{3}{2}(4 \pi)^{2 / 3} N^{-1 / 3}+\left(\frac{N-1}{N}\right) \frac{3}{2}(2 \pi)^{2 / 3} N^{2 / 3}-\frac{1}{2}(2 \pi)^{2} r^{2} \\
& \quad \triangleq d_{0} .
\end{aligned}
$$

Lemma 14. For $\partial \Lambda_{+}=\left\{q \in W^{1,2}\left(R / Z, R^{2}\right) \mid q(t+1 / N)=\right.$ $R(N) q(t), \exists 1 \leq \widetilde{i}_{0} \leq N, \widetilde{t}_{0} \in[0,1]$ s.t. $\left.q_{\tilde{i}_{0}}\left(\widetilde{t}_{0}\right)=q\left(\widetilde{t}_{0}\right)\right\}$, we have

$$
\begin{aligned}
& \inf _{q \in \partial \Lambda_{+}} f(q) \\
& \quad \geq \frac{3}{2}(2 \pi)^{2 / 3} N^{1 / 3}+\left(\frac{N-1}{N}\right) \frac{3}{2}(2 \pi)^{2 / 3} N^{2 / 3}-\frac{1}{2}(2 \pi)^{2} r^{2} \\
& \triangleq d_{1} .
\end{aligned}
$$

Proof. Similar to the proof of Lemma 13, Lemma 14 holds.

Proof of Theorem 2. In order to get Theorem 2, we are going to find a test loop $\widetilde{q} \in \Lambda_{-}$such that $f(\widetilde{q})=d_{2}$. Then the minimizer of $f$ on $\bar{\Lambda}_{-}$must be a noncollision solution if $d_{2}<$ $d_{0}$.

Let $a>0, b>0, \theta \in[0,2 \pi)$ and

$$
\tilde{q}-q_{N}=(a \cos (-2 \pi t+\theta), b \sin (-2 \pi t+\theta))^{T} .
$$

Hence,

$$
\begin{gathered}
\tilde{q}-q_{1}=\left(r \cos 2 \pi t-r \cos \left(2 \pi t+\frac{2 \pi}{N}\right)\right. \\
\quad+a \cos (-2 \pi t+\theta), r \sin 2 \pi t \\
\left.-r \sin \left(2 \pi t+\frac{2 \pi}{N}\right)+b \sin (-2 \pi t+\theta)\right)^{T}, \\
\quad \vdots \\
\tilde{q}-q_{i}=\left(r \cos 2 \pi t-r \cos \left(2 \pi t+\frac{i}{N} 2 \pi\right)\right. \\
\quad+a \cos (-2 \pi t+\theta), r \sin 2 \pi t \\
\left.\quad-r \sin \left(2 \pi t+\frac{i}{N} 2 \pi\right)+b \sin (-2 \pi t+\theta)\right)^{T},
\end{gathered}
$$

$$
\begin{aligned}
\widetilde{q}-q_{N-1}=(r & \cos 2 \pi t-r \cos \left(2 \pi t+\frac{N-1}{N} 2 \pi\right) \\
& +a \cos (-2 \pi t+\theta), r \sin 2 \pi t \\
& \left.-r \sin \left(2 \pi t+\frac{N-1}{N} 2 \pi\right)+b \sin (-2 \pi t+\theta)\right)^{T} .
\end{aligned}
$$


It is easy to see that $\tilde{q} \in \Lambda_{-}$and

$$
\begin{aligned}
\left|\dot{\tilde{q}}-\dot{q}_{1}\right|^{2}=(2 \pi)^{2}\{ & \frac{a^{2}+b^{2}}{2}-\frac{a^{2}-b^{2}}{2} \cos (4 \pi t-2 \theta) \\
& +r^{2}\left(2-2 \cos \frac{2 \pi}{N}\right)-(a+b) r \\
& \times\left[\cos (4 \pi t-\theta)-\cos \left(4 \pi t+\frac{2 \pi}{N}-\theta\right)\right] \\
& \left.+(a-b) r\left[\cos \theta-\cos \left(\frac{2 \pi}{N}+\theta\right)\right]\right\},
\end{aligned}
$$

$$
\begin{aligned}
\left|\widetilde{q}-q_{1}\right|=\{ & \frac{a^{2}+b^{2}}{2}+\frac{a^{2}-b^{2}}{2} \cos (4 \pi t-2 \theta) \\
& +r^{2}\left(2-2 \cos \frac{2 \pi}{N}\right)+(a+b) r \\
& \times\left[\cos (4 \pi t-\theta)-\cos \left(4 \pi t+\frac{2 \pi}{N}-\theta\right)\right] \\
& \left.+(a-b) r\left[\cos \theta-\cos \left(\frac{2 \pi}{N}+\theta\right)\right]\right\}^{1 / 2},
\end{aligned}
$$

$$
\begin{aligned}
\left|\dot{\vec{q}}-\dot{q}_{i}\right|^{2}=(2 \pi)^{2}\{ & \frac{a^{2}+b^{2}}{2}-\frac{a^{2}-b^{2}}{2} \cos (4 \pi t-2 \theta) \\
& +r^{2}\left(2-2 \cos \frac{i}{N} 2 \pi\right)-(a+b) r \\
& \times\left[\cos (4 \pi t-\theta)-\cos \left(4 \pi t+\frac{i}{N} 2 \pi-\theta\right)\right] \\
& \left.+(a-b) r\left[\cos \theta-\cos \left(\frac{i}{N} 2 \pi+\theta\right)\right]\right\},
\end{aligned}
$$

$$
\begin{aligned}
\left|\widetilde{q}-q_{i}\right|= & \left\{\frac{a^{2}+b^{2}}{2}+\frac{a^{2}-b^{2}}{2} \cos (4 \pi t-2 \theta)\right. \\
& +r^{2}\left(2-2 \cos \frac{i}{N} 2 \pi\right)+(a+b) r \\
& \times\left[\cos (4 \pi t-\theta)-\cos \left(4 \pi t+\frac{i}{N} 2 \pi-\theta\right)\right] \\
& \left.+(a-b) r\left[\cos \theta-\cos \left(\frac{i}{N} 2 \pi+\theta\right)\right]\right\}^{1 / 2},
\end{aligned}
$$

$$
\left|\dot{\tilde{q}}-\dot{q}_{N}\right|^{2}=(2 \pi)^{2}\left(\frac{a^{2}+b^{2}}{2}-\frac{a^{2}-b^{2}}{2} \cos (4 \pi t-2 \theta)\right),
$$

$$
\begin{gathered}
\left|\widetilde{q}-q_{N}\right|=\sqrt{\frac{a^{2}+b^{2}}{2}+\frac{a^{2}-b^{2}}{2} \cos (4 \pi t-2 \theta),} \\
\left|\dot{q}_{1}\right|^{2}=\cdots=\left|\dot{q}_{N}\right|^{2}=(2 \pi)^{2} r^{2} .
\end{gathered}
$$

Therefore by (36)-(42), we get

$f(\tilde{q})$

$$
\begin{aligned}
= & \frac{1}{N} \int_{0}^{1} \sum_{i=1}^{N}\left[\frac{1}{2}\left|\dot{\tilde{q}}-\dot{q}_{i}\right|^{2}+\frac{N}{\left|\widetilde{q}-q_{i}\right|}\right] d t-\frac{1}{2 N} \int_{0}^{1} \sum_{i=1}^{N}\left|\dot{q}_{i}\right|^{2} d t \\
= & \left(a^{2}+b^{2}\right) \pi^{2}+(2 \pi)^{2} r^{2} \\
& +2 \pi^{2}(a-b) r\left[\cos \theta-\frac{1}{N} \sum_{i=1}^{N} \cos \left(\frac{i}{N} 2 \pi+\theta\right)\right] \\
& +\sum_{i=1}^{N} \int_{0}^{1}\left\{\frac{a^{2}+b^{2}}{2}+\frac{a^{2}-b^{2}}{2} \cos (4 \pi t-2 \theta)\right. \\
& +r^{2}\left(2-2 \cos \frac{i}{N} 2 \pi\right)+(a+b) r \\
& \quad\left[\cos (4 \pi t-\theta)-\cos \left(4 \pi t+\frac{i}{N} 2 \pi-\theta\right)\right] \\
& \left.+(a-b) r\left[\cos \theta-\cos \left(\frac{i}{N} 2 \pi+\theta\right)\right]\right\}^{-1 / 2} d t \\
& -\frac{1}{2}(2 \pi)^{2} r^{2} \\
= & 2 \pi^{2}\left(\frac{a^{2}+b^{2}}{2}+r^{2}\right) \\
& +\sum_{i=1}^{N} \int_{0}^{1}\left\{\frac{a^{2}+b^{2}}{2}+\frac{a^{2}-b^{2}}{2} \cos (4 \pi t-2 \theta)\right. \\
& +2 \pi^{2}(a-b) r\left[\cos \theta-\frac{1}{N} \sum_{i=1}^{N} \cos \left(\frac{i}{N} 2 \pi+\theta\right)\right] \\
& \left.+(4 \pi t-\theta)-\cos \left(4 \pi t+\frac{i}{N} 2 \pi-\theta\right)\right]
\end{aligned}
$$

In order to estimate $d_{2}$, we have computed the numerical values of $d_{2}=f(q)$ over some selected test loops. The computation of the integral that appears in (43) has been done using the function \{quad\} of Mathematica 7.1 with an error less than $10^{-6}$. The results of the numerical explorations are given in Table 1. 
TABle 1: Parameters of test loops for Theoerm 2.

\begin{tabular}{|c|c|c|c|c|c|}
\hline$N$ & $a$ & $b$ & $\theta$ & $d_{0}$ & $d_{2}$ \\
\hline 2 & 0.11 & 0.29 & $\pi / 2$ & 9.813298 & 9.753899 \\
\hline 2 & 0.13 & 0.25 & $\pi$ & 9.813298 & 9.753243 \\
\hline 2 & 0.13 & 0.27 & $\pi$ & 9.813298 & 9.643849 \\
\hline 2 & 0.15 & 0.21 & $\pi$ & 9.813298 & 9.790068 \\
\hline 2 & 0.15 & 0.23 & $\pi / 5$ & 9.813298 & 9.746803 \\
\hline 2 & 0.17 & 0.19 & $\pi$ & 9.813298 & 9.751237 \\
\hline 2 & 0.17 & 0.21 & $\pi / 5$ & 9.813298 & 9.625193 \\
\hline 2 & 0.19 & 0.17 & $\pi$ & 9.813298 & 9.784859 \\
\hline 2 & 0.19 & 0.19 & $\pi$ & 9.813298 & 9.564603 \\
\hline 2 & 0.21 & 0.21 & $\pi$ & 9.813298 & 9.279445 \\
\hline 2 & 0.23 & 0.15 & $\pi$ & 9.813298 & 9.793205 \\
\hline 2 & 0.23 & 0.23 & $\pi / 5$ & 9.813298 & 9.109854 \\
\hline 2 & 0.25 & 0.13 & $\pi / 2$ & 9.813298 & 9.730925 \\
\hline 2 & 0.25 & 0.25 & $\pi / 5$ & 9.813298 & 9.038706 \\
\hline 3 & 0.15 & 0.67 & $\pi / 30$ & 11.523843 & 11.505860 \\
\hline 3 & 0.15 & 0.69 & $\pi / 30$ & 11.523843 & 11.444212 \\
\hline 3 & 0.17 & 0.65 & $\pi / 30$ & 11.523843 & 11.493238 \\
\hline 3 & 0.17 & 0.67 & $\pi / 20$ & 11.523843 & 11.452135 \\
\hline 3 & 0.17 & 0.69 & $\pi / 20$ & 11.523843 & 11.400124 \\
\hline 3 & 0.19 & 0.63 & $\pi / 30$ & 11.523843 & 11.519350 \\
\hline 3 & 0.19 & 0.65 & $\pi / 20$ & 11.523843 & 11.455969 \\
\hline 3 & 0.19 & 0.67 & $\pi / 20$ & 11.523843 & 11.386608 \\
\hline 3 & 0.61 & 0.23 & $\pi$ & 11.523843 & 11.516685 \\
\hline 3 & 0.63 & 0.19 & $\pi$ & 11.523843 & 11.489791 \\
\hline 3 & 0.63 & 0.21 & $\pi$ & 11.523843 & 11.436105 \\
\hline 3 & 0.65 & 0.17 & $\pi$ & 11.523843 & 11.461786 \\
\hline 3 & 0.65 & 0.19 & $\pi$ & 11.523843 & 11.392115 \\
\hline 3 & 0.65 & 0.21 & $\pi$ & 11.523843 & 11.349366 \\
\hline 3 & 0.67 & 0.15 & $\pi$ & 11.523843 & 11.472422 \\
\hline 3 & 0.67 & 0.17 & $\pi$ & 11.523843 & 11.383978 \\
\hline 3 & 0.67 & 0.19 & $\pi$ & 11.523843 & 11.324970 \\
\hline 3 & 0.69 & 0.13 & $\pi$ & 11.523843 & 11.522980 \\
\hline 3 & 0.69 & 0.15 & $\pi$ & 11.523843 & 11.412094 \\
\hline 3 & 0.69 & 0.17 & $\pi$ & 11.523843 & 11.334189 \\
\hline
\end{tabular}

For the parameters $a, b$, and $\theta$ given in Table 1 , we all have $d_{2}<d_{0}$. For $4 \leq N \leq 1000$, we do not find suitable parameters $a \in[0.01,2 r), b \in[0.01,2 r)$, and $\theta=$ $\pi, \pi / 2, \pi / 3, \ldots, \pi / 29, \pi / 30$ such that $d_{2}<d_{0}$, where $r$ is the radius of the first $N$ bodies with equal masses. Hence we only consider the case $N=2,3$. This completes the proof of Theorem 2.

Proof of Theorem 4. To get Theorem 4, we are going to find a test loop $\bar{q} \in \Lambda_{+}$such that $f(\bar{q})=d_{3}$. Then the minimizer of $f$ on $\bar{\Lambda}_{+}$must be a noncollision solution if $d_{3}<d_{1}$.

Let $a>0, \theta \in[0,2 \pi)$, and

$$
\bar{q}-q_{N}=a e^{\sqrt{-1} 2 \pi t} e^{\sqrt{-1} \theta} .
$$

Hence,

$$
\begin{aligned}
\bar{q}-q_{1} & \\
& =q_{N}+a e^{\sqrt{-1} 2 \pi t} e^{\sqrt{-1} \theta}-q_{1} \\
& =r e^{\sqrt{-1} 2 \pi t}\left(1-e^{\sqrt{-1}(2 \pi / N)}\right)+a e^{\sqrt{-1} 2 \pi t} e^{\sqrt{-1} \theta}, \\
& \vdots \\
\bar{q}-q_{i} & \\
= & q_{N}+a e^{\sqrt{-1} 2 \pi t} e^{\sqrt{-1} \theta}-q_{i} \\
& =r e^{\sqrt{-1} 2 \pi t}\left(1-e^{\sqrt{-1}(i / N) 2 \pi}\right)+a e^{\sqrt{-1} 2 \pi t} e^{\sqrt{-1} \theta}, \\
& \vdots \\
\bar{q}-q_{N-1} & \\
= & q_{N}+a e^{\sqrt{-1} 2 \pi t} e^{\sqrt{-1} \theta}-q_{N-1} \\
= & r e^{\sqrt{-1} 2 \pi t}\left(1-e^{\sqrt{-1}((N-1) / N) 2 \pi}\right)+a e^{\sqrt{-1} 2 \pi t} e^{\sqrt{-1} \theta} .
\end{aligned}
$$

It is easy to see that $\bar{q} \in \Lambda_{+}$and

$$
\begin{aligned}
& \left|\dot{\bar{q}}-\dot{q}_{1}\right|^{2}=(2 \pi)^{2}\left\{r^{2}\left(2-2 \cos \frac{2 \pi}{N}\right)+a^{2}\right. \\
& \left.+2 \operatorname{ar}\left[\cos \theta-\cos \left(\theta-\frac{2 \pi}{N}\right)\right]\right\}, \\
& \left|\bar{q}-q_{1}\right|=\left\{r^{2}\left(2-2 \cos \frac{2 \pi}{N}\right)+a^{2}\right. \\
& \left.+2 \operatorname{ar}\left[\cos \theta-\cos \left(\theta-\frac{2 \pi}{N}\right)\right]\right\}^{1 / 2}, \\
& \left|\dot{\bar{q}}-\dot{q}_{i}\right|^{2}=(2 \pi)^{2}\left\{r^{2}\left(2-2 \cos \frac{i}{N} 2 \pi\right)+a^{2}\right. \\
& \left.+2 \operatorname{ar}\left[\cos \theta-\cos \left(\theta-\frac{i}{N} 2 \pi\right)\right]\right\}, \\
& \left|\bar{q}-q_{i}\right|=\left\{r^{2}\left(2-2 \cos \frac{i}{N} 2 \pi\right)+a^{2}\right. \\
& \left.+2 a r\left[\cos \theta-\cos \left(\theta-\frac{i}{N} 2 \pi\right)\right]\right\}^{1 / 2}, \\
& \left|\dot{\bar{q}}-\dot{q}_{N}\right|^{2}=(2 \pi)^{2} a^{2}, \quad\left|\bar{q}-q_{N}\right|=a, \\
& \left|\dot{q}_{1}\right|^{2}=\cdots=\left|\dot{q}_{N}\right|^{2}=(2 \pi)^{2} r^{2} .
\end{aligned}
$$


Therefore by (48)-(53), we get

$f(\bar{q})$

$$
\begin{aligned}
= & \frac{1}{N} \int_{0}^{1} \sum_{i=1}^{N}\left[\frac{1}{2}\left|\dot{\bar{q}}-\dot{q}_{i}\right|^{2}+\frac{N}{\left|\bar{q}-q_{i}\right|}\right] d t \\
& -\frac{1}{2 N} \int_{0}^{1} \sum_{i=1}^{N}\left|\dot{q}_{i}\right|^{2} d t \\
= & 2 \pi^{2} a^{2}+(2 \pi)^{2} r^{2} \\
& +(2 \pi)^{2}\left[\operatorname{arcos} \theta-\frac{a r}{N} \sum_{i=1}^{N} \cos \left(\theta-\frac{i}{N} 2 \pi\right)\right] \\
& +a^{-1}+\sum_{i=1}^{N-1}\left\{r^{2}\left(2-2 \cos \frac{i}{N} 2 \pi\right)+a^{2}\right. \\
= & d_{3}(a, \theta) \cdot a^{-1}+\sum_{i=1}^{N-1}\left\{r^{2}\left(2-2 \cos \frac{i}{N} 2 \pi\right)+a^{2}\right. \\
& \left.-\frac{1}{2}(2 \pi)^{2} r^{2} \quad+2 a r\left[\cos \theta-\cos \left(\theta-\frac{i}{N} 2 \pi\right)\right]\right\}^{-1 / 2} \\
& +2 \pi^{2}\left(a^{2}+r^{2}\right) \\
& (2 \pi)^{2}\left[\operatorname{arcos} \theta-\frac{a r}{N} \sum_{i=1}^{N} \cos \left(\theta-\frac{i}{N} 2 \pi\right)\right]
\end{aligned}
$$

In order to estimate $d_{3}$, we have computed the numerical values of $d_{3}=f(q)$ over some selected test loops. The results of the numerical explorations show that by choosing some $a \in[0.11,12.50]$ and $\theta=\pi$, one has $d_{3}<d_{1}$ for $2 \leq N \leq 32402$. From the computational data, let $\theta=\pi, \pi / 2, \pi / 3, \ldots, \pi / 19, \pi / 20$, and we find that $d_{3}<d_{1}$ holds for $2 \leq N \leq 19$ with some $a \in[0.11,0.90]$. But for $20 \leq N \leq 32402$, no matter how we choose the value of $a \in[0.11,12.50]$, we obtain $d_{3}<d_{1}$ only if $\theta=\pi$. For $N=32403$, the computational data shows that $d_{3} \gg d_{1}$ for all $a \in[0.11,10.00]$ and $\theta=$ $\pi, \pi / 2, \pi / 3, \ldots, \pi / 99, \pi / 100$. Then combined with Figure 1 , it is easy to see that a.e. $d_{3}>d_{1}$ for all $a \in[0.11,12.50]$ and $\theta=$ $\pi, \pi / 2, \pi / 3, \ldots, \pi / 99, \pi / 100$. The same holds for $N=32404$ from Figure 2 and the computational data. Moreover, we can get the same conclusions for $32405 \leq N \leq 100000$. Hence, we only consider the case $2 \leq N \leq 32402$. This completes the proof of Theorem 4 .

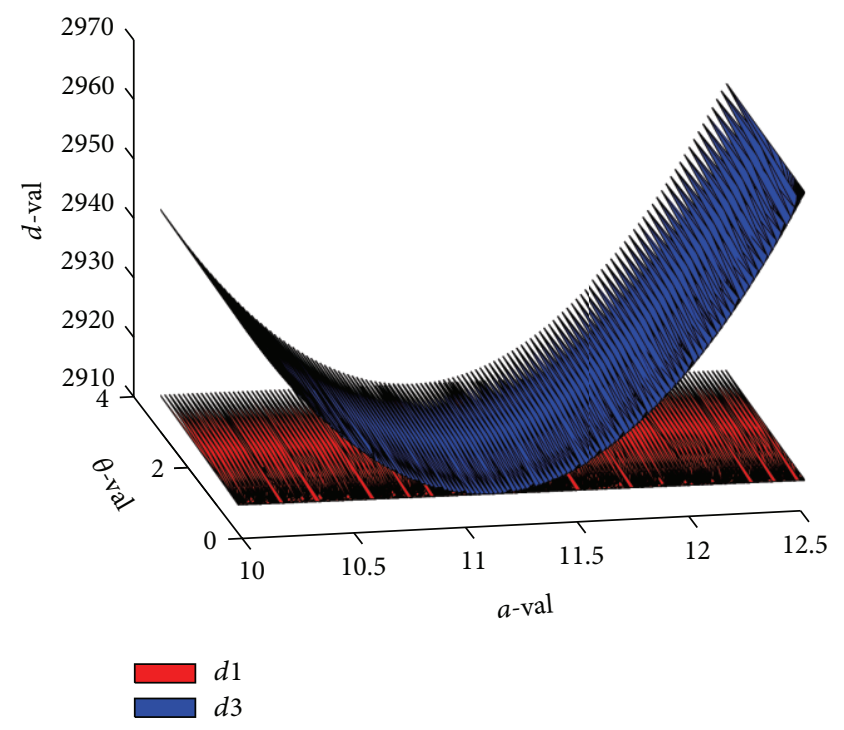

Figure 1: $N=32403$.

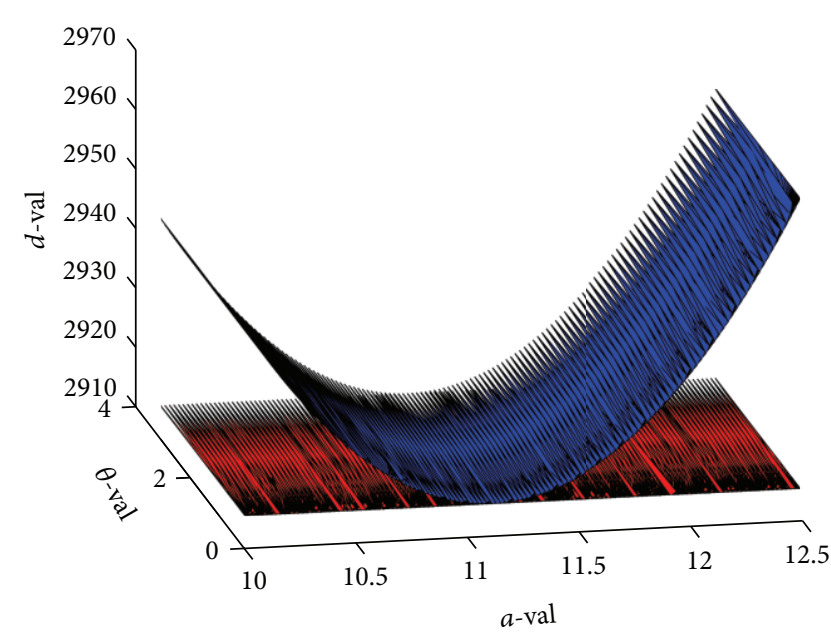

FIGURE 2: $N=32404$

\section{Acknowledgments}

This study is supported by the national natural Science Foundation of China. The authors would like to thank Professor Shiqing Zhang, Taisong Xiong, Donglun Wu and Yiyang Deng for some helpful discussions, and the referee for his/her many valuable comments and suggestions on this paper.

\section{References}

[1] A. Chenciner and R. Montgomery, "A remarkable periodic solution of the three-body problem in the case of equal masses," Annals of Mathematics, vol. 152, no. 3, pp. 881-901, 2000.

[2] A. Chenciner, "Action minimizing solutions of the Newtonian $n$-body problem: from homology to symmetry," in Proceedings 
of the International Mathematical Union (ICM '02), pp. 255-264, Beijing, China, 2002.

[3] A. Venturelli, "Une caractérisation variationnelle des solutions de Lagrange du problème plan des trois corps," Comptes Rendus de l'Académie des Sciences, vol. 332, no. 7, pp. 641-644, 2001.

[4] C. Marchal, "How the method of minimization of action avoids singularities," Celestial Mechanics \& Dynamical Astronomy, vol. 83, no. 1-4, pp. 325-353, 2002.

[5] C. Moore, "Braids in classical dynamics," Physical Review Letters, vol. 70, no. 24, pp. 3675-3679, 1993.

[6] C. Simó, "New families of solutions in $n$-body problems," Progress in Mathematics, vol. 201, pp. 101-115, 2001.

[7] D. L. Ferrario and S. Terracini, "On the existence of collisionless equivariant minimizers for the classical $N$-body problem," Inventiones Mathematicae, vol. 155, no. 2, pp. 305-362, 2004.

[8] F. Y. Li, S. Q. Zhang, and X. X. Zhao, "Periodic solutions for spatial restricted N+1-body problems," Preprint.

[9] S. Zhang and Q. Zhou, "Symmetric periodic noncollision solutions for $n$-body-type problems," Acta Mathematica Sinica, vol. 11, no. 1, pp. 37-43, 1995.

[10] S. Q. Zhang and Q. Zhou, "A minimizing property of Lagrangian solutions," Acta Mathematica Sinica, vol. 17, no. 3, pp. 497-500, 2001.

[11] S. Zhang and Q. Zhou, "Variational methods for the choreography solution to the three-body problem," Science in China A, vol. 45, no. 5, pp. 594-597, 2002.

[12] S. Zhang and Q. Zhou, "Nonplanar and noncollision periodic solutions for $n$-body problems," Discrete and Continuous Dynamical Systema A, vol. 10, no. 3, pp. 679-685, 2004.

[13] S. Zhang, Q. Zhou, and Y. Liu, "New periodic solutions for 3body problems," Celestial Mechanics \& Dynamical Astronomy, vol. 88, no. 4, pp. 365-378, 2004.

[14] U. Bessi and V. Coti Zelati, "Symmetries and noncollision closed orbits for planar $n$-body-type problems," Nonlinear Analysis, vol. 16, no. 6, pp. 587-598, 1991.

[15] X. X. Zhao and S. Q. Zhang, "Periodic solutions for circular restricted 4-body problems with Newtonian potentials," Preprint.

[16] J. Mawhin and M. Willem, Critical Point Theory and Hamiltonian Systems, vol. 74, Springer, New York, NY, USA, 1989.

[17] M. Struwe, Variational Methods, Springer, Berlin, Germany, 3rd edition, 1990.

[18] R. S. Palais, “The principle of symmetric criticality," Communications in Mathematical Physics, vol. 69, no. 1, pp. 19-30, 1979.

[19] W. B. Gordon, "A minimizing property of Keplerian orbits," The American Journal of Mathematics, vol. 99, no. 5, pp. 961-971, 1977.

[20] Y. Long and S. Zhang, "Geometric characterizations for variational minimization solutions of the 3-body problem," Acta Mathematica Sinica, vol. 16, no. 4, pp. 579-592, 2000. 


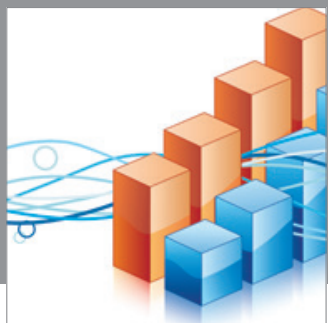

Advances in

Operations Research

mansans

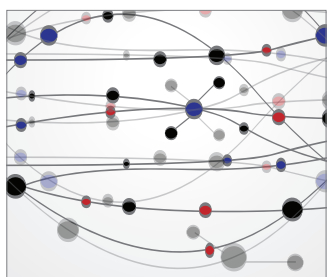

The Scientific World Journal
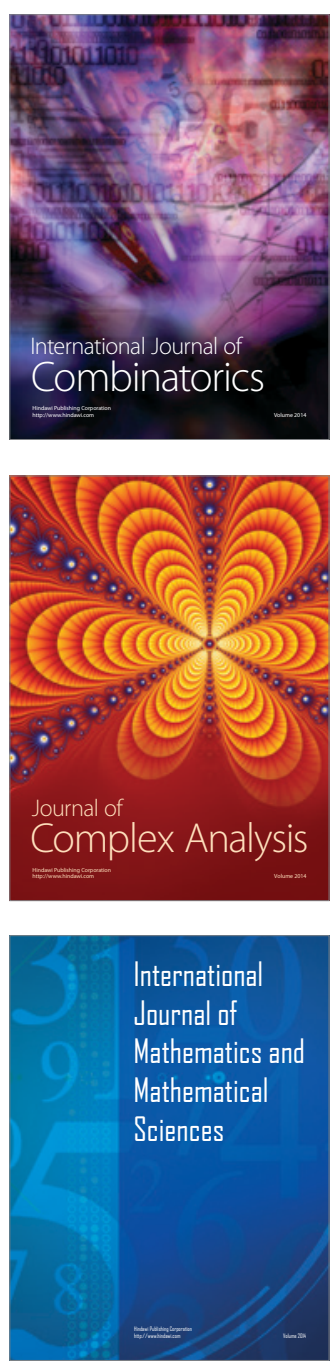
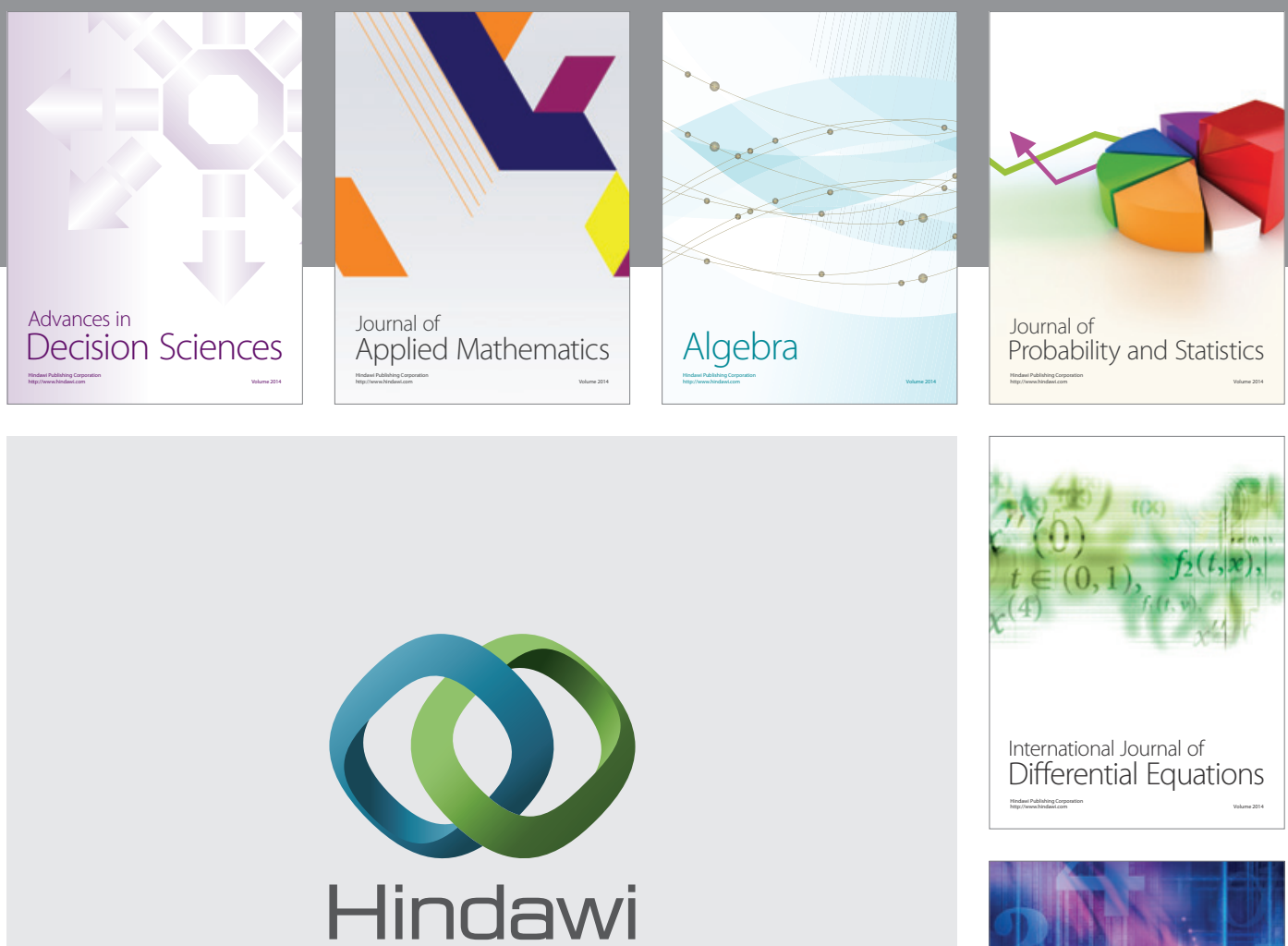

Submit your manuscripts at http://www.hindawi.com
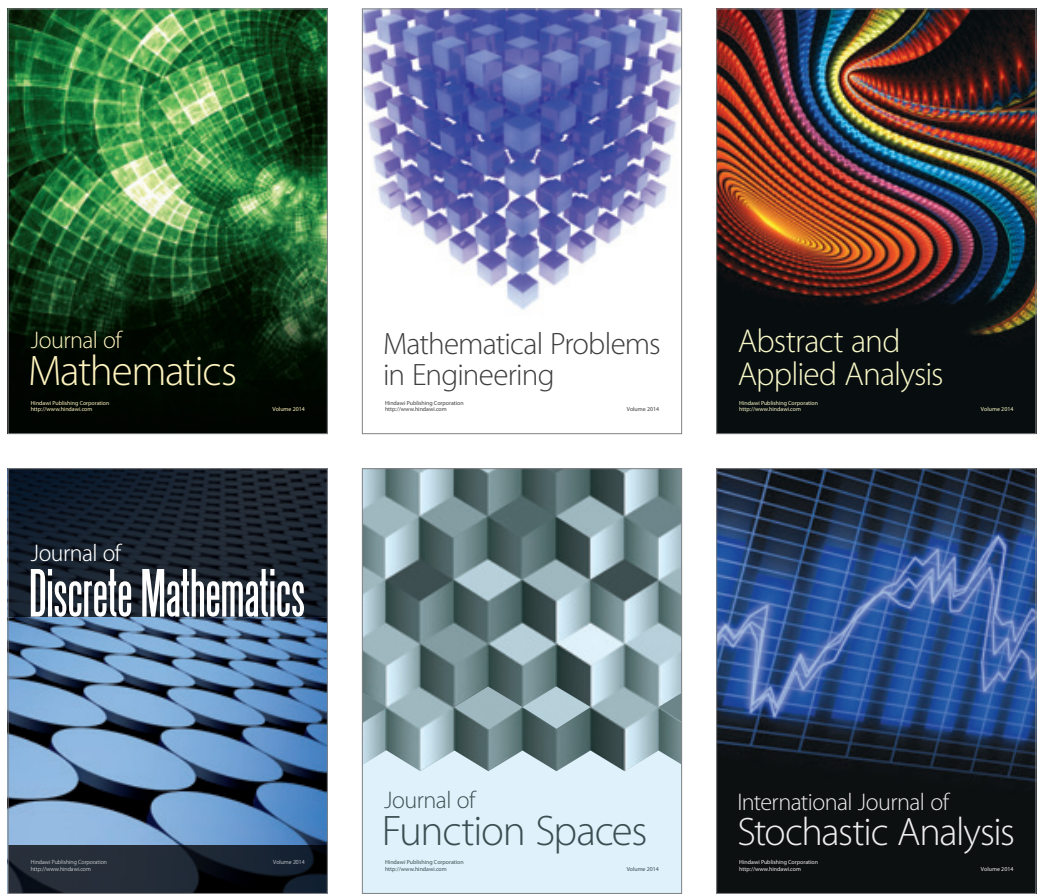

Journal of

Function Spaces

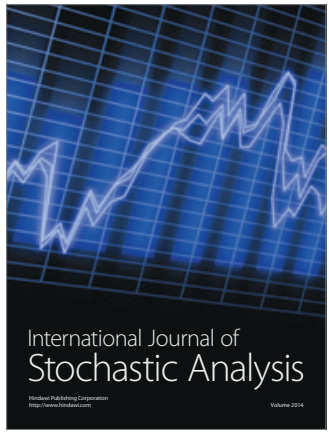

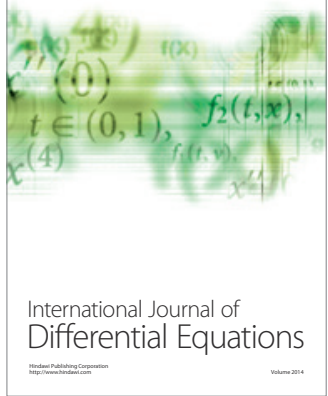
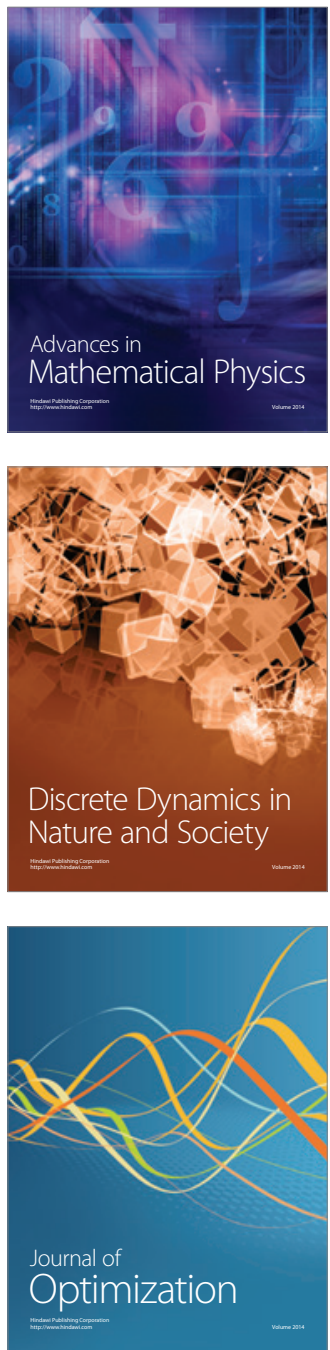